Digital Press Social Sciences and Humanities

Le niveau d'équivalence de l'interjection dans Spirou et Fantasio et sa traduction en Indonésien

Agiska Tanesya and Myrna Laksman-Huntley

Proceeding of Conférence internationale sur le français 2018

Joesana Tjahjani, Merry Andriani, Sajarwa, Wening Udasmoro (eds) 


\title{
Le niveau d'équivalence de l'interjection dans Spirou et Fantasio et sa traduction en indonésien
}

\author{
Agiska Tanesya* et Myrna Laksman-Huntley \\ Département de Français, Faculté des Sciences Humaines, Universitas Indonesia, Depok, Indonesia \\ *e-mail : agiska.tanesya@ui.ac.id
}

\section{Résumé}

Cet article traite du niveau de correspondance de la traduction d'interjections françaises en indonésien dans la bande dessinée Spirou et Fantasio et sa traduction. À l'aide de méthodes qualitatives et de techniques d'étude de la littérature, les données ont été analysées basé sur la théorie de l'analyse des composantes de Leech (1981) et celle de l'équivalence de Williams (2013). Les types des écarts etaient analysées en utilisant la théorie du décalage de Catford (1965). Les résultats montrant plus d'équivalence totale indiquent que les traducteurs maintiennent la correspondance et les messages même si les interjections en français sont plus variées qu'en indonésien. Cependant, l'équivalent de la traduction des interjections en indonésien est jugé plus approprié à la fonction dans le contexte des images qu'en français. L'interjection en forme onomatopique consistant généralement en une collection de voyelles ou de combinaisons de consonnes tend à soutenir les expressions et les sentiments des personnages. Ce type d'interjection domine d'autres formes d'interjection. Bien que la traduction de l'onomatopée en indonésien ne soit faite que par la répétition des traductions précédentes, l'onomatopée utilisée a des significations différentes selon le contexte. Comparé à d'autres bandes dessinées, l'humour de genre Spirou et Fantasio est plus susceptible de faire apparaître l'histoire dans l'image, de sorte que les interjections et les phrases simples ont senti tout à fait favorable à l'image. L'utilisation de phrases simples dans cette bande dessinée indique qu'aucune forme de changement n'est trouvée autre que le déplacement du mot classe dans la traduction indonésienne.

\section{Mots-clés}

correspondence en traduction, l'écart en traduction, la traduction, bande dessinée Spirou et Fantasio

\begin{abstract}
This article deals with the level of correspondence of the translations of French interjections into Indonesian in the comics Spirou et Fantasio and its translation. Using qualitative methods and literature techniques, data were analyzed based on Leech's (1981) component analysis theory and Williams' equivalence theory (2013). Types of shape changes were analyzed using Catford's (1965) shift theory. The results that show more total equivalence indicate that translators maintain correspondence and messages even though French interjections are more varied than in Indonesian. However, the translation equivalent of interjections in Indonesian is considered more appropriate for the function in the context of images than in French. The onomatopic-type interjection usually consists solely of a collection of vowels or consonantal combinations serves to support the expressions and feelings of the characters. This type of interjection dominates other forms of interjection. Although the translation of onomatopoeia into Indonesian is done only by the repetition of previous translations, the onomatopoeia that is used has different meanings depending on the context. Compared to other comics, genre humor Spirou and Fantasio is more likely to bring up the story in the picture, so the interjections and simple sentences felt quite favorable to the picture. The use of simple sentences in this comic indicates that there is no change of form is found other than the displacement of the word class in Indonesian translation
\end{abstract}

\section{Keywords}

correspondence, change of form, translation, Spirou and Fantasio comics 


\section{Introduction}

Selon McCloud, comics sont des images et des symboles positionnés dans un certain ordre pour fournir des informations et un moyen de communication pour obtenir des réponses esthétiques des lecteurs (2001). En Europe, comics est connue sous le nom de bande dessinée (BD). Celle-ci suscite toujours la controverse, elle est donc qualifiée de dangereuse par les critiques de bandes dessinées (Miller, 2007).

Lancé à partir de la page du site Dupuis, Spirou et Fantasio est l'une des bandes dessinées franco belges populaires publiés par Dupuis qui apparut en 1948 avec des genres d'aventure et de sciencefiction. Spirou et Fantasio a été largement traduit en plusieurs langues, dont l'indonésien par Agustina en 2010 a été publié par PT Elex Media Komputindo.

Cette bande dessinée a différentes histoires dans un livre. Une série se compose de quatre histoires différentes. Selon le site de Dupuis, l'histoire présentée semble facile à comprendre car l'âge de lecture de ce livre est de 9 ans. Le langage utilisé pour le dialogue a tendance à être des phrases simples et non complexes. Voici une comparaison de l'utilisation de la langue dans les bandes dessinées Spirou et Fantasio, Tintin, et Schtroumpf.
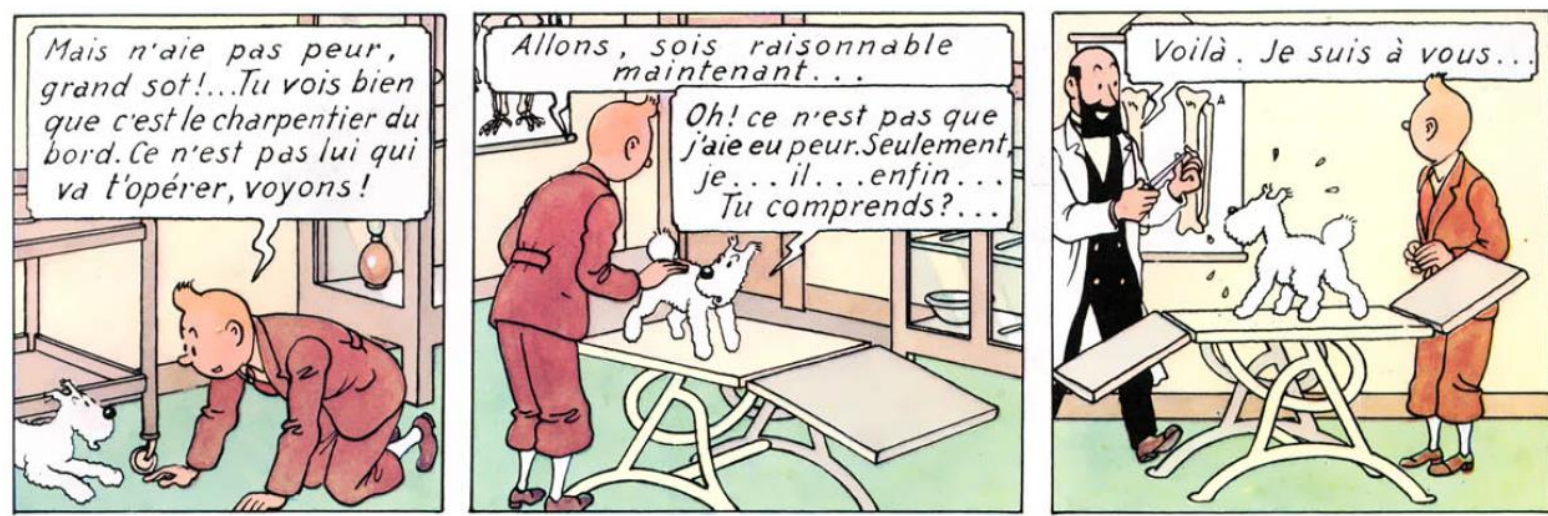

Figure 1 Tintin au Congo (1946)
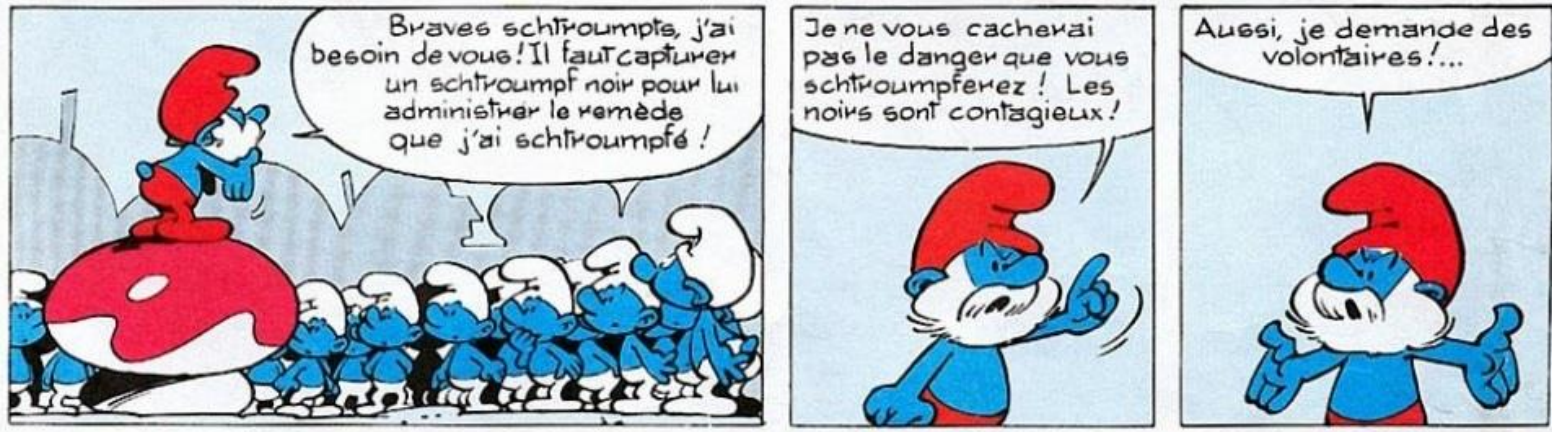

Figure 2 Les Schtroumpfs Noirs (1959)
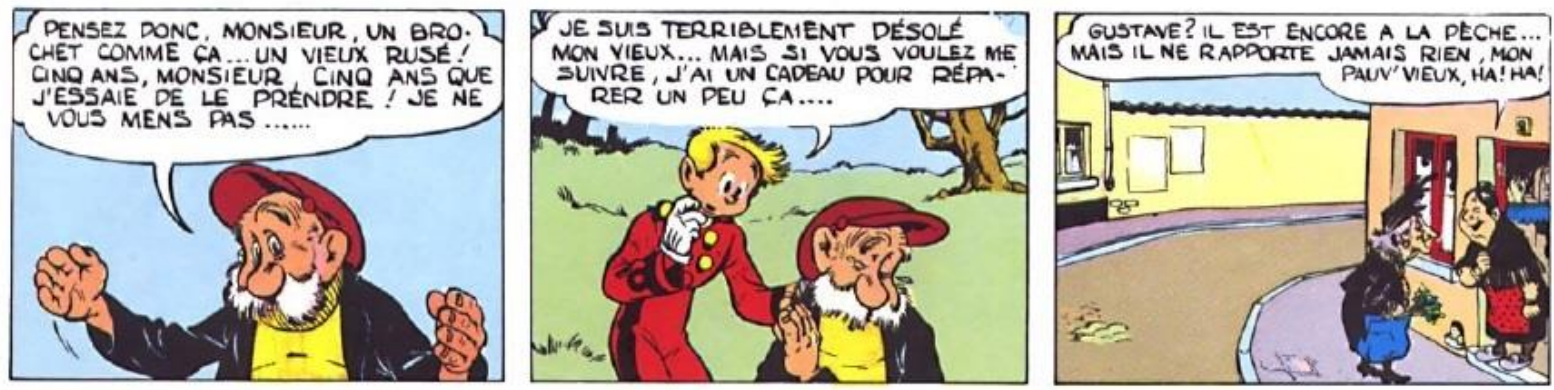

Figure 3 Spirou et Fantasion (1950)

À partir des trois images présentées ci-dessus, on peut voir que l'utilisation des phrases et verbes dans la bande dessinée (BD) Spirou et Fantasio est plus simple et ne contient aucun élément compliqué ni difficile. 
Les BDs sont différentes des autres œuvres littéraires telles que les contes. Si les contes sont de longs récits, les BDs utilisent des images et dialoguent directement. Dans les BDs, le dialogue devient l'un des éléments qui jouent un rôle important dans la relance de l'histoire. Généralement, le dialogue contient des interjections pour transmettre les sentiments et les intentions d'un contexte dans l'image. L'interjection sert d'exclamation dans un discours pour exprimer des sentiments, des pensées, des invitations et des appels.

Selon Kridalaksana (1994), en traduisant les interjections, un traducteur éprouve souvent des difficultés à déterminer de bon équivalent. Cela est dû au fait que l'interjection syntaxique ne se rapporte pas aux autres mots des énoncés, de sorte qu'elle peut entraîner des modifications dans la traduction, telles que des modifications du niveau d'équivalence, les écarts de classes de mots et le sens. Les changements de traduction peuvent être causées par des différences entre la langue source (LS) et la langue cible (LC) associées aux racines de la langue, la grammaire, et au point de vue culturelle des locuteurs. Selon Catford (1965), la traduction est remplacement des éléments de texte de de LS avec ceux selon LC. Pour obtenir une correspondance appropriée entre LS et LC, il y a souvent un écart. Ainsi, on peut dire que, dans le processus de traduction, un traducteur doit modifier le contenu textuel de LS avec celui de LC qui est comparable.

La culture est l'un des facteurs qui peuvent être un obstacle à la traduction. Cela peut arriver parce que chaque communauté a ses propres caractéristiques et il n'y a pas de même culture. L'un des éléments de la culture est le style de langage incorporé dans le type de texte. La coutume et le style de langue deviennent souvent des obstacles dans la traduction (Nida, 1982). L'interjection peut être assurée non seulement par des médias oraux, mais aussi par des médias écrits, ainsi que dans des dialogues sur des BDs et des romans. La France et l'Indonésie sont deux pays qui ont les caractéristiques de leurs cultures respectives. Bien sûr, il est difficile pour le traducteur de trouver une correspondance appropriée en raison des différences culturelles entre les deux pays dans la traduction des interjections.

Il y a eu beaucoup d'études utilisant les BDs comme corpus. L'une d'entre elles est une thèse de Tetuko, Programme d'études françaises 2014, Universitas Indonesia. Cette étude traite de l'équivalence des expressions phatiques du français en indonésien dans la BD d'Astérix. Tetuko a utilisé la théorie de la forme équivalente et l'alignement de forme de Catford (1965) et trouvé 53 expressions phatiques composées de 28 qui fonctionnent pour établir la communication et 25 pour mettre fin à la communication. Dans une autre thèse de recherche précédente est celle de Cahyonowati, de l'Universitas Indonesia en 2000; elle a étudié un roman pour examiner les interjections en utilisant la théorie de la traduction de Catford (1965), Nida et Taber (1982), et Newmark (1988).

\subsection{Interjection en français et en indonésien}

Dans la 14ème édition du Bon Usage publié en 2007, Grevisse mentionne l'interjection comme un cri lancé dans un discours pour exprimer des sentiments, des pensées, des commandes, des invitations et des appels. Selon Grevisse, l'interjection est généralement courte, composée d'une syllabe et suivie d'un point d'exclamation. Basé de sa forme, Grevisse distingue l'interjection en cinq types : les onomatopées (Ah ! Eh ! Oh!), les noms (Attention! Courage!), les adjectifs (Bon! Chic! Mince), les adverbes (Bien! Ben !), les verbes (Gare ! Halte! Tiens !), et les phrases complètes (Nom d'un tonnerre!). De plus, Grevisse a également expliqué que le sens généré par l'interjection dépend du ton, de l'accent, du mouvement, de l'attitude du locuteur et du contexte.

Cependant, Kridalaksana (1994) explique que l'interjection est interprétée comme un mot utilisé pour exprimer les sentiments de ses interlocuteurs. Contrairement à Grevisse, Kridalaksana divise les interjections en deux formes : les formes de base (aduh, aduhai, ayo) et les formes dérivées qui proviennent généralement de mots indonésiens à la fois standard et non standard ou des fragments d'appels arabes (Alhamdulillah, syukur, astaga, buset).

\subsection{Analyse de la signification des composants}

Leech (1981) décrit la sémantique que l'étude du sens rôle très important dans le processus de communication qui implique deux personnes ou plus et il faut comprendre ce qui est prononcé. Selon Leech, ce qui signifie l'analyse des composants peut être effectuée par l'analyse componentielle en utilisant un signe binaire $(+)$ ou $(-)$ pour chaque composante du sens traduit. 


\subsection{L'équivalence en traduction par Williams}

Selon Catford (dans Cahyonowati, 2000), l'équivalence est une situation dans laquelle LS et LC produisent le même message. Williams (2013) divise le niveau d'équivalence en quatre niveaux : l'équivalence totale, l'équivalence facultative, l'équivalence approximative et l'équivalence nulle. Premier, l'équivalence totale se produit lorsque le mot dans LS a une signification et une fonction complètes qui correspondent à l'équivalence. Deuxième, l'équivalence facultative se produit lorsque le traducteur a plus d'une possibilité équivalente dans LS. Troisième, l'équivalence approximative se produit lorsqu'il n'y a pas de mot dans LS qui correspond au mot dans LC. Quatrième, l'équivalence nulle se produit lorsqu'il n'y a pas de mot dans LC qui correspond au mot dans LS.

\subsection{Les écarts en traduction par Catford}

Les écarts se produisent souvent afin d'obtenir un équivalent raisonnable et conformément aux règles de Catford (1965), cité dans Cahyonowati (2000). Il y a deux types d'écart, à savoir l'écart de catégorie et l'écart de niveau. Les écarts de catégories traitent des écarts intra-systèmes, de la structure, de la classe, et d'unité.

a. La structure de décalage est liée à la structure de la phrase, à la clause ou au cadre.

b. La classe se produit lorsqu'une classe indique que LS est transféré à une autre classe de mots dans LC.

c. L'unité qui se produit depuis l'unité syntaxique est supérieur à l'unité inférieure ou vice versa.

d. Les écarts intra-systèmes se produisent en raison des différences de systèmes internes entre LS et LC.

L'écart de niveau est un écart au niveau de la phonologie, de la morphologie, de la grammaire et du lexique.

\section{Méthode}

Cette étude décrit le problème du niveau d'équivalence et de l'écart de forme dans la traduction des bandes dessinées Spirou et Fantasio : Quatre Aventures de Spirou et Fantasio. Le corpus est en version digital sur les pages du site http://love-freebooks.blogspot.co.id/2016/12/a-venir-spirou-et-fantasiocollection.html?m=1. Cette BD de Franquin a été traduite par Pratama et publié en 2010 par PT Elex Media Komputindo. Les chercheurs ont également examiné des images pour ajuster l'interaction avec le contexte. Pour trouver des correspondances de traduction et d'alignement modifiant la forme d'interjections français en indonésien dans les bandes dessinées, nous utilisons des méthodes de recherche qualitative selon Creswell (2010) à l'étude des techniques de la littérature. Des mots contenant des interjections dans les bandes dessinées françaises et indonésiennes ont été collectées, classifiées et comparées pour l'analyse en utilisant Le Petit Robert micro en 2013 et le grand dictionnaire en langue indonésienne (KBBI, 2016) accessible sur la page du site https://kbbi.kemdikbud.go.id. Nous pouvons donc trouver un élément de sens harmonieux et approprié pour voir la comparabilité de l'interprétation des interjections entre langue française (LF) et langue indonésienne (LI).

\section{Discussion}

328 interjections ont été réalisées, composées de deux niveaux d'équivalence différents, 260 interjections en équivalence totale et 68 interjections de l'équivalence approximative.

\subsection{L'équivalence totale}

Le niveau d'équivalence totale dans la traduction des interrogations dans les BDs de Spirou et Fantasio et la traduction peuvent être visualisés à partir des résultats de l'analyse suivante. 

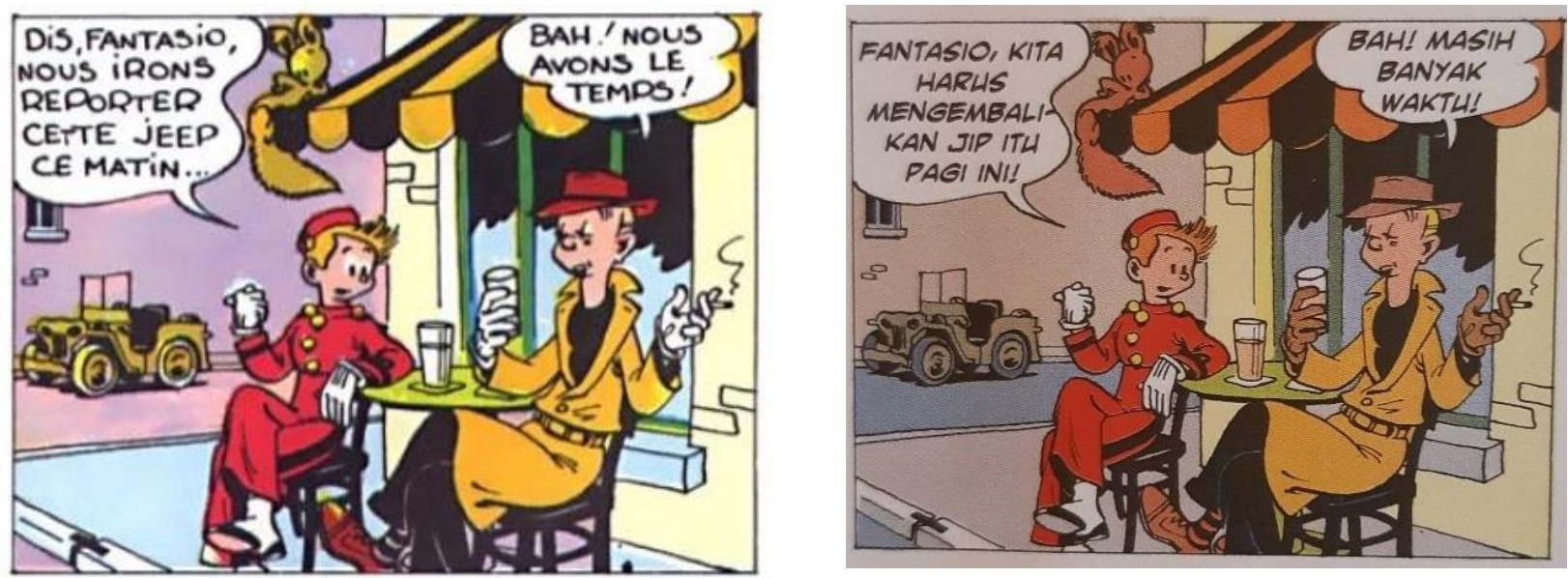

Figure 4 Spirou et Fantasio (l'équivalence totale)

\section{LF : Bah! Nous avons le temps ! \\ LI : Bah! Masih banyak waktu!}

Les citations de dialogue ci-dessus sont un morceau de dialogue entre Spirou et Fantasio. Le contexte dans la phrase est quand Spirou et Fantasio se détendent sur le porche de la maison. Spirou a invité Fantasio à rendre la jeep qu'ils ont empruntée, mais Fantasio a refusé et a estimé qu'ils pourraient le rendre plus tard.

Tableau 1 L'analyse de la signification des composants entre Bah et Bah

\begin{tabular}{|l|l|l|}
\hline & Bah & Bah \\
\hline L'ignorance & + & + \\
\hline L'indifférence & + & + \\
\hline Le dégoût & + & + \\
\hline
\end{tabular}

Basé sur l'analyse de la signification des composants entre Bah et Bah, les deux ont des significations complètement similaires, à savoir l'ignorance, l'indifférence, le dégoût. On peut voir à travers le tableau ci-dessus que les deux n'ont pas de composants du sens différents. De cette façon, on peut dire que l'interjection Bah et Bah ont un niveau d'équivalence totale.

Dans ces images, les composants qui conviennent à l'appel du Bah et Bah ne sont que des significations d'ignorance et d'indifférence. Les résultats de l'analyse de la composante du sens entre Bah et Bah qui ont été décrits ci-dessus montrent que la signification produite a une équivalence totale lorsqu'elle est vue également avec le contexte sur l'image. Soutenu par les gestes et les mouvements de la main de Fantasio. L'équivalent utilisé par les traducteurs pour interjection en indonésien est Bah. L'interprétation en langue indonésienne utilisée a une fonction conforme à la situation et au contexte de l'image.

\subsection{L'équivalence approximative}

68 interjections avec un niveau d'équivalence approximative se basent sur les résultats de l'analyse suivante. 


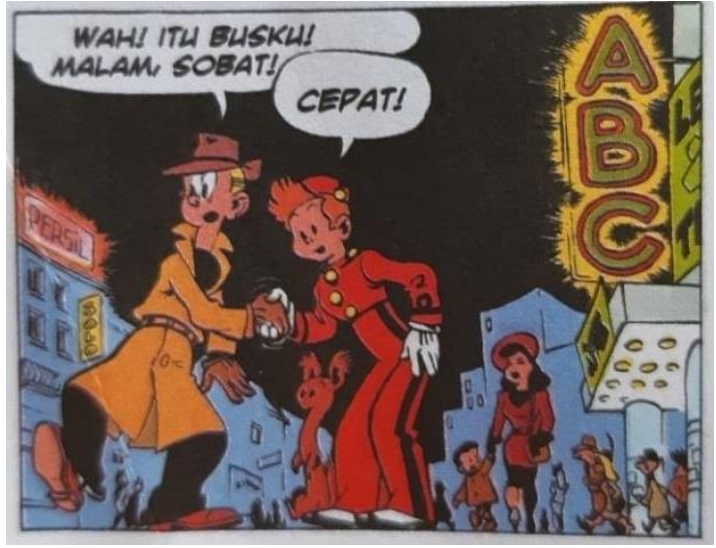

Figure 5 Fantasio et Spirou (L'équivalence approximative)

LF : Holà ! Voici mon bus ! Soir, vieux !

LI : Wah, itu busku! Malam, sobat!

Les citations ci-dessus sont un morceau de dialogue entre Fantasio et Spirou. Le contexte contenu dans le dialogue de citation ci-dessus est lorsque Spirou et Fantasio doivent se séparer parce qu'il fait sombre.

Tableau 2 L'analyse de la signification des composants entre Holà ! et Wah !

\begin{tabular}{|l|l|l|}
\hline & Holà ! & Wah! \\
\hline L'appel & + & - \\
\hline La déception & - & + \\
\hline L'admiration & - & + \\
\hline Le choc & + & + \\
\hline L'etonnement & - & + \\
\hline
\end{tabular}

Basé sur des résultats de l'analyse de la signification des composants ci-dessus, on peut voir que le mot Holà et Wah n'ont qu'un seul sens qui est aligné entre d'autres significations, à savoir le choc. Le mot Wah a le sens de déception, d'admiration et d'étonnement dans LI, alors que le mot Holà n'a pas de sens harmonieux autre que le choc. On peut dire que le mot Holà et Wah ont un niveau d'équivalence approximative parce qu'il y a des significations différentes. En examinant la table l'analyse de la signification des composants, les deux interjections ne contiennent qu'un seul même sens, à savoir le choc.

S'il est ajusté dans le contexte de l'image, le geste de Fantasio semble être pressé après avoir vu l'arrivée du bus. Il a également été vu à travers l'expression de Fantasio qui a été choqué quand il a vu le bus est arrivé. De cette façon, la signification générée dans l'interjection Holà et Wah est le choc. L'équivalent utilisé par le traducteur pour l'interjection en indonésien est Wah. L'interjection Wah a une fonction correspondant à la situation de l'image par rapport à Holà en français. Bien qu'il ait un composant le choc, mais le mot Holà est utilisé dans la langue française plus approprié pour saluer que l'identification des sentiments de surprise.

Après avoir analysé toutes les interventions dans les BDs, il y avait un niveau d'équivalence totale et approximative. Cependant, le niveau équivalence totale est plus élevé par rapport à l'équivalence approximative. À en juger par l'utilisation interjection onomatopée, l'interjection en français est plus variée que l'indonésienne. Interjection en indonésien souvent utilise la répétition de mots, comme dans interjection l'appel ou cherche d'attention. En français, il y avaient interjection 'tiens, holà et salut' alors que en indonésienne il n'y a que le mot Hei.

Le niveau de l'équivalence totale qui domine dans la traduction de interjections prouve que la traductrice souhaite maintenir l'équivalence selon la fonction. Basé sur la théorie de Williams (2013) en ce qui concerne le degré d'équivalence dans la traduction, si on trouve que la signification et la fonction des interjections de LS et LC sont compatibles, les deux ont de niveau d'équivalence totale. Cependant, s'il y a des différences dans les composants mais que les fonctions produites sont les mêmes, elles ont un niveau d'équivalence approximative. 


\subsection{Les écarts de la classe de mots en traduction}

Nous avons également analysé les écarts dans la traduction des interjections du français vers l'indonésienne. L'écart trouvé dans les BDs et sa traduction ne fait que déplacer la classe de mots. Dans ces BDs, cinq interjections qui subissent des écarts de la classe de mots dans la traduction de l'interjection dans les BDs peut être vu à partir des résultats de l'analyse suivante.

Tableau 3 Résultats de l’analyse

\begin{tabular}{|l|l|l|}
\hline Nom $\rightarrow$ verbe & Nom $\rightarrow$ adjectif & Nom $\rightarrow$ adverbia \\
\hline Au secours - tolong & Stop - gawat & Halte - berhenti \\
\hline du calme - tenanglah & & Non - jangan \\
\hline
\end{tabular}

Quatre des cinq interjections des BDs Spirou et Fantasio : Quatre Aventures de Spirou et Fantasio, qui ont connu un écart dans la classe de mots, sont impératives. L'interjection prend la forme d'un ordre pour une phrase ou un verbe qui énonce une interdiction ou une nécessité de faire quelque chose. Après avoir analysé l'alignement des formes dans les BDs, on a trouvé un écart au niveau de la classe de mots qui se produit en basculant la classe de mots de la LF vers une autre classe de mots. On peut dire que l'écart a été effectué par la traductrice pour obtenir et fournir une équivalence raisonnable et harmonieuse entre LF et LI. L'écart de classe de mots qui apparaît en équivalence totale entre les interjections LF et LI qui ont été analysées à l'aide de la table de signification Leech est nécessaire pour obtenir la correspondance appropriée et ne modifie pas le message de LS et LC.

L'existence d'un niveau d'équivalence totale et approximative ainsi que les écarts constatés dans la traduction des interjections du français en indonésien dans les bandes dessinées étudiées ont confirmé la théorie de la traduction de Catford. La capacité de trouver l'équivalence en phase pour obtenir la traduction acceptable et l'écart qui apparaissent dans la traduction d'interjection prouve que le matériel de texte en langue source et langue cible présentent des différences fondées sur le système linguistique et deux cultures différentes.

\section{Conclusion}

Basé des résultats de la recherche effectuée, on peut conclure que, pour déterminer le sens de l'interjection, le contexte dans une image est très important pour comprendre la situation de l'histoire. La relation entre les images et le dialogue influence largement les lecteurs à comprendre la réaction des personnages dans les bandes dessinées. De cette manière, on peut dire que les images et les interjections fonctionnent toutes les deux pour obtenir le message et comprendre un but et acceptable pour le lecteur. L'interjection en tant que forme d'expression exprimée par les personnages doit être vue dans le contexte de la phrase ou de celle qui suit, de sorte que le sens trouvé soit lié au sens de l'interprétation. Bien que l'interjection ait le même mode d'écriture et d'expression, le sens peut varier. Vu de l'équivalence du sens, a trouvé plus d'équivalence totale qu'approximative dans les résultats de la traduction.

L'équivalence des significations produites en français et indonésien montre que l'interjection dans les deux langues contient une composante harmonisée de la pleine signification. L'équivalence approximative trouvée a eu lieu parce qu'il y avait un composant différent de la signification. Malgré cela, la signification résultante contient toujours l'équivalence de la langue source. De plus, les résultats obtenus après l'analyse de l'alignement des formes dans l'interprétation des interjections ont révélé des modifications du niveau de la classe de mots. L'écart de la classe de mots nominaux à l'adjective, les verbes, et aux adverbes se produisent parce que l'interjection en français obtient le mot indonésien équivalent d'une classe de mots différente.

Le matériel du texte de LS sous la forme d'interjections traduites en LC subit un niveau différent d'équivalence et les écarts dans la classe de mots prouvent que la traductrice tente d'ajuster l'équivalent dans le texte. En effet, la traductrice doit conserver et ne modifie pas le message et l'intention du rédacteur de texte LS lors de la traduction. Si le message de l'auteur LS n'est pas bien transmis dans LC, le lecteur aura sûrement du mal à comprendre la signification du texte LS. De cette façon, les écarts, l'équivalence approximative ou les traductions totales sont nécessaires pour parvenir à une harmonie dans la traduction.

Pour susciter l'empathie des lecteurs, l'interjection dans les bandes dessinées est utilisée afin qu'elle puisse être emportée par l'atmosphère de l'histoire. De plus, les lecteurs peuvent facilement accepter le message et l'intention sur les bandes dessinées de Spirou et Fantasio, de sorte que l'interjection soit très 
favorable pour la livraison des sentiments des personnages de l'histoire. L'équivalent en indonésien utilisé a tendance à être plus adapté à sa fonction lorsqu'il est lié au contexte et à la situation de l'image par rapport à l'utilisation des interjections en français. Les résultats de cette étude sont différents des études précédentes sur les interjections dans les traductions de romans français. Parce que les romans ne sont pas supportés par des images, on doit mener la traduction de interjections avec une forme similaire et avec l'aide des phrases qui suivent. Ainsi, les écarts de forme dans une traduction d'un roman se produit plus au niveau de l'unité et de la classe de mots. On peut donc dire que les BDs sont différents des autres œuvres littéraires. Les traducteurs peuvent facilement trouver des harmonies correspondantes grâce à l'aide des images disponibles plutôt qu'aux phrases qui les suivent par rapport aux romanx qui ne présentent qu'un long récit.

\section{Références}

Cahyonowati, R. (2000). Tinjauan Perpadanan Interjeksi Bahasa Prancis dan Bahasa Indonesia dalam

Novel Madame Bovary dan Terjemahannya: Nyonya Bovary. (mémoire de bachelor). Universitas Indonesia, Depok.

Catford, J. C. (1965). A Linguistic Theory of Translation. London: Oxford University Press.

Creswell, J. W. (2010). Research Design: Qualitative, Quantitative, and Mixed Methods Approaches. London: SAGE Publications.

Franquin, A. (2010). Spirou et Fantasio. (D. A. Pratama, Trans.). Paris: Dupuis. Retrieved from

https://www.dupuis.com/seriebd/UK/spirou-and-fantasio-by-franquin/3068

Grevisse, M., \& Goose, A. (2007). Le Bon Usage (14th ed.). Paris: Duculot.

KBBI. (2016). KBBI Daring. Retrieved from https://kbbi.kemdikbud.go.id

Kridalaksana, H. (1994). Kelas Kata dalam bahasa Indonesia (2nd ed.). Jakarta: PT. Gramedia Pustaka Utama.

Leech, G. (1981). Semantics: The Study of Meaning. London: Penguin.

McCloud, S. (2001). Understanding Comics: The Invisible Arts. (Kinanti, Trans.). Jakarta: PT. Gramedia Pustaka Utama.

Miller, A. (2007). Reading Bande Dessinée: Critical Approaches to French-Language Comic Strip. Chicago: Intellect Book.

Nida, E. A., \& Taber, C. R. (1982). The Theory and Practice of Translation. Leiden: Brill.

Robert, P., Rey, A., \& Rey-Debove, J. (2013). Le Robert Micro. Paris: Le Robert.

Tetuko, B. (2014). Penerjemahan Ungkapan Fatis Bahasa Prancis ke dalam Bahasa Indonesia dalam

Komik Astérix. (mémoire de bachelor). Universitas Indonesia, Depok.

Williams, J. (2013). Theories of Translations. London: Palgrave Macmillan. 